\title{
Cytostatic factor inactivation is induced by a calcium-dependent mechanism present until the second cell cycle in fertilized but not in parthenogenetically
} activated mouse eggs

\author{
Magdalena Zernicka-Goetz ${ }^{1,2}$, Maria A. Ciemerych ${ }^{1}$, Jacek Z. Kubiak ${ }^{2}$, Andrzej K. Tarkowski ${ }^{1}$ \\ and Bernard Maro ${ }^{2, *}$ \\ ${ }^{1}$ Department of Embryology, Institute of Zoology, University of Warsaw, Krakowskie Przedmiescie 26/28, 00-927 Warsaw 64, \\ Poland \\ 2Département de Biologie du Développement, Institut Jacques Monod, CNRS - Université Paris VII, Tour 43-2, Place Jussieu, \\ 75005 Paris, France
}

*Author for correspondence

\section{SUMMARY}

Cytostatic factor (CSF) is an activity responsible for the metaphase II arrest in vertebrate oocytes. This activity maintains a high level of maturation promoting factor (MPF) in the oocyte and both activities are destroyed after fertilization or parthenogenetic activation. To study some of the characteristics of the mechanism involved in MPF and CSF destruction, we constructed hybrid cells between metaphase II arrested oocytes and early embryos obtained after fertilization or artificial activation. We found that the behavior of hybrid cells differed depending upon the type of oocyte activation. Initially, the reaction of both types of hybrid cells was similar, the nuclear envelope broke down and chromatin condensation was induced. However, while metaphase II oocytes fused with parthenogenetic eggs remained arrested in M-phase, the oocytes fused with fertilized eggs underwent activation and passed into interphase. This ability of fertilized eggs to induce oocyte activation was still present at the beginning, but not at the end of the second embryonic cell cycle. Oocyte activation induced by fusion with a fertilized egg could be prevented when calcium was chelated by BAPTA. Thus, element(s) of the mechanism involved in calcium release triggered by a sperm component at fertilization remain(s) active until the second cell cycle and is (are) inactivated before the end of the 2-cell stage.

Key words: mouse oocyte, CSF, cytostatic factor, MPF, maturation promoting factor, cell cycle, sperm, parthenogenesis

\section{INTRODUCTION}

Ovulated mouse oocytes remain arrested at the second meiotic metaphase (metaphase II), until fertilization or parthenogenetic activation. Meiotic arrest is maintained by an activity called cytostatic factor (CSF; for review see Masui, 1991). CSF was first discovered in frog eggs by transferring cytoplasm from metaphase II arrested oocytes to one blastomere at the 2-cell stage leading to an M-phase arrest of the injected blastomere while the non-injected blastomere continued to cleave (Masui and Markert, 1971). In the mouse oocyte, fusion experiments were used to demonstrate the presence of an activity similar to CSF (Kubiak et al., 1993). To maintain the M-phase arrest, CSF prevents the inactivation of the M-phase promoting factor (MPF; Masui and Markert, 1971). MPF is an heterodimer of cdc2 kinase and cyclin B that is required for the resumption of meiotic maturation and progression through meiosis to metaphase II (for review see Norbury and Nurse, 1992). At the exit from M-phase, cyclin degradation leads to MPF inactivation. In the mouse oocyte, the metaphase II arrest can be maintained for several hours during which there is a balanced synthesis and degradation of cyclin B, the regulatory subunit of active MPF, and this equilibrium seems to be regulated at least partially by CSF (Kubiak et al., 1993). Three protein kinases have been shown to be involved in CSF activity, the product of the protooncogene c-mos (Sagata et al., 1989), cdk2 (Gabrielli et al., 1993) and MAP kinase (Haccard et al., 1993). The nature of the molecular interactions between CSF and MPF are still unclear, although it has been shown that cyclin degradation is prevented in cell-free extracts from Xenopus oocytes containing CSF. One of the most striking properties of CSF is that it is degraded after MPF (Lorca et al., 1991; Watanabe et al., 1991; Weber et al., 1991). This observation seems to be in contradiction with the definition given originally by Masui (1991), where CSF was defined as an activity that should be destroyed to allow MPF destruction. However, our recent data (Kubiak et al., 1993) suggest that CSF acts more like a reversible enzymatic inhibitor, decreasing the efficiency of the cyclin degradation machinery either by modifying cyclin and making its degradation more difficult or by inhibiting directly the cyclin degradation enzyme. This inhibitory effect would 
be overcome at oocyte activation by bringing in an activator of the cyclin degradation machinery. Finally, CSF destruction only seems to be possible in interphase at the time of pronuclear formation (Kubiak, 1989; Zernicka-Goetz, 1991). The destruction of MPF and CSF at fertilization takes place following a release of calcium from intracellular stores and involves the calmodulin dependent protein kinase II (Lorca et al., 1993). In mouse oocytes, fertilization triggers a series of calcium spikes lasting several (3-4) hours (Cuthbertson et al., 1981; Kline and Kline, 1992). Parthenogenetic activation, via a variety of stimuli (for review see Kaufman, 1983), also induces an increase in intracellular calcium concentration (Cuthbertson et al., 1981; Cuthbertson and Cobbold, 1985; Kline and Kline, 1992; Vincent et al., 1992). With most parthenogenetic stimuli, however, there is only a single calcium transient rather than the series of spikes observed after sperm entry (for example see Winston et al., 1994). The respective timings of inactivation of MPF (as measured by H1 kinase activity) and CSF (as measured by c-mos destruction) are similar (Weber et al., 1991) regardless of whether there is a single, or a series of calcium spikes. MPF activity reappears during each mitotic M-phase, but it is not blocked by CSF, which is characteristic for meiosis in vertebrates. To study some of the characteristics of the mechanism involved in MPF and CSF destruction, we fused metaphase II arrested oocytes and early embryos obtained after fertilization or artificial activation and followed the fate of CSF activity in these hybrid cells.

\section{MATERIALS AND METHODS}

\section{Collection and culture of oocytes and embryos}

F1 (CBA/HxC57B1/10) female mice were superovulated by injections of $5 \mathrm{IU}$ of pregnant mare's serum gonadotrophin (PMSG, Intervet) and $5 \mathrm{IU}$ of human chorionic gonadotrophin (hCG, Intervet) 48-52 hours apart. Metaphase II oocytes were collected 13-14 hours after hCG injection. Fertilized 1- and 2-cell embryos were obtained from mated females between 21 and 44 hours after hCG. All oocytes and embryos were collected and cultured in M2 medium supplemented with $4 \mathrm{mg} / \mathrm{ml}$ BSA (Whittingham, 1971) under paraffin oil at $37^{\circ} \mathrm{C}$. Cumulus cells were removed by treatment with 300 units hyaluronidase/ml of phosphate-buffered saline (PBS) and the zona pellucida with $0.5 \%$ pronase (Sigma) in PBS.

\section{Parthenogenetic activation}

Metaphase II oocytes (17-18 hours after hCG) were treated with $8 \%$ ethanol (Merck) in M2 medium for 8 minutes at room temperature (Cuthbertson, 1983) and then washed carefully. The activated oocytes were cultured for the next 12-14 hours before they were used for fusion. The majority of artificially activated eggs were haploid, but diploid parthenogenetically activated eggs were also observed.

\section{Fusions}

Metaphase II oocytes were agglutinated with different experimental partners in phytohaemagglutinin (PHA; Sigma) diluted in M2 without BSA at a final concentration of $300 \mu \mathrm{g} / \mathrm{ml}$ for 5 minutes on $1 \%$ agarose at $37^{\circ} \mathrm{C}$. The cell pairs (or trios in the case of 2-cell embryos), were then exposed to a $45 \%$ solution of polyethylene glycol (PEG, molecular mass: 2000, Fluka) in BSA-free M2 for 60-70 seconds to induce fusion. Then, the cells were washed carefully in M2 + BSA, and cultured further at $37^{\circ} \mathrm{C}$.

\section{Experimental variants}

Metaphase II oocytes were fused with:

(a) early 1-cell fertilized eggs (21-24 hours post hCG) in G1/S;

(b) late 1-cell fertilized eggs (27-29 hours post hCG) in G2;

(c) early 2-cell embryos (34-36 hours post hCG) in G1/S;

(d) late 2-cell embryos (46 hours post hCG) in G2;

(e) late 1-cell haploid parthenogenetically activated eggs (12-14 hours after activation) in G2;

(f) late 1-cell diploid parthenogenetically activated eggs (12-14 hours after activation) in $\mathrm{G} 2$;

(g) metaphase II oocytes.

\section{Chelation of intracellular calcium}

Intracellular calcium was chelated by incubation of eggs immediately after PEG treatment with $10 \mu \mathrm{M}$ 5,5-dimethyl-BAPTA-AM (Molecular Probes), which after entering the cell is hydrolysed to BAPTA and buffers elevations of intracellular calcium without changing the basal level (Kline and Kline, 1992). This concentration of BAPTA blocks calcium transients effectively without affecting embryo viability (Winston et al., 1995).

\section{Examination of hybrid cells}

The hybrid cells were examined for signs of fusion every 10-15 minutes during the first hour after PEG treatment and every hour during the following 3-6 hours of culture. Hybrid cells that lysed $(15 \%)$ after exposure to PEG were discarded. Fused and unfused cell pairs, as well as control embryos, were cultured for different times varying from 3 to 24 hours after PEG treatment. Cytological studies were carried out using whole-mount preparations stained with hematoxylin (Tarkowski, 1966). In addition, the microtubules and nuclei were observed by immunofluorescence. The fixation and labelling of oocytes were performed as described previously (Maro et al., 1984), and for tubulin staining we used the rat monoclonal antibody YL1/2 specific for tyrosinated tubulin and a fluorescein-conjugated anti-rat secondary antibody (Miles). The chromatin was stained with propidium iodide $(5 \mu \mathrm{g} / \mathrm{ml}$; Molecular Probes). Samples were observed using a Bio-Rad MRC-600 laser scanning confocal microscope.

\section{RESULTS}

To perform our studies, we needed a fusion method that did not itself induce oocyte activation and provided large numbers of hybrids. These requirements led us to use the polyethylene glycol method (Spindle, 1981) instead of the electrofusion technique used in our previous study (Kubiak et al., 1993). To exclude the possibility that the fusion procedure itself induced oocyte activation we fused metaphase II-arrested oocytes with each other, and found that only $15 \%$ (4/27) of hybrid cells were activated after 24 hours of culture (Table 1, line 1 and Fig. 1A). Using this method, we then examined the fate of metaphase II oocytes fused with late 1-cell parthenogenetically activated eggs in the G2 phase (12-14 hours after ethanol treatment). The majority of hybrids that formed arrested in Mphase for at least 24 hours. In total, 88\% (36/41) of these hybrid cells remained blocked in M-phase until the end of culture (Table 1, line 2 and Fig. 1B) and most of them (70\%) contained one common metaphase spindle. Since control parthenogenetic eggs (treated with PEG) underwent the first cleavage division (Fig. 1C), the metaphase arrest of hybrids containing a parthenogenetic component could not be due to the inability of parthenogenotes to cleave under our culture conditions. 

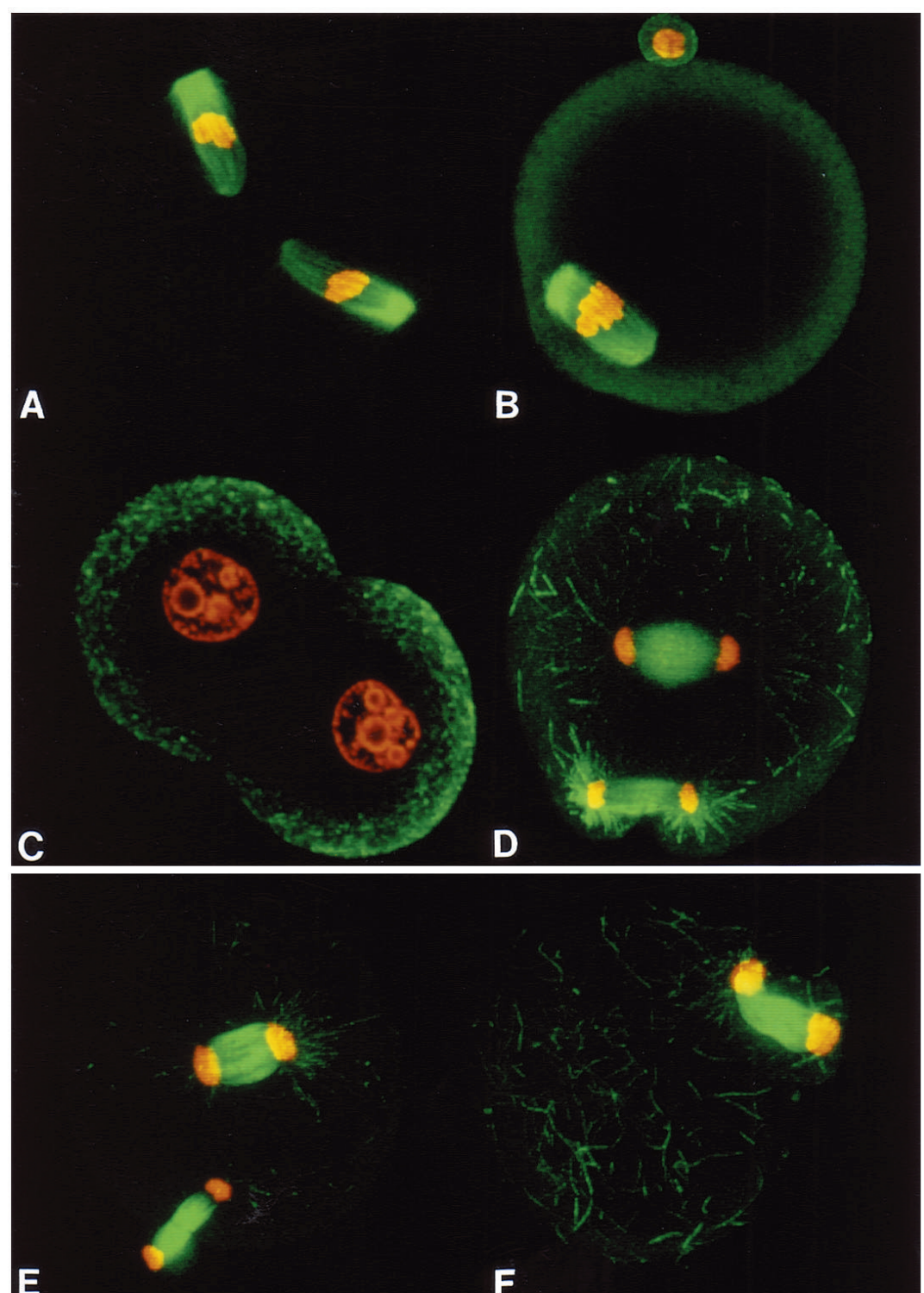

E

$\mathbf{F}$

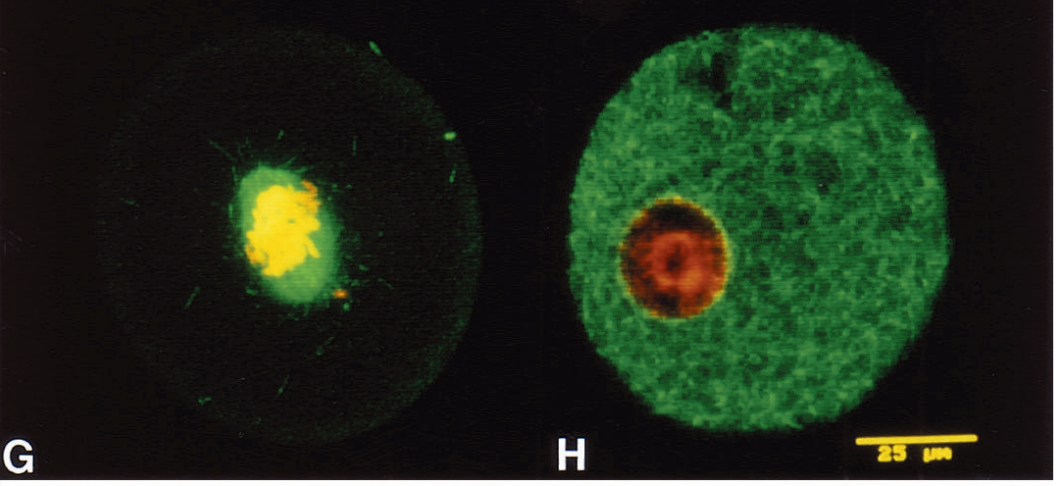

Fig. 1. Morphology of hybrids obtained after fusion of: (A) two fused metaphase II oocytes - hybrid arrested in M-phase, observed 24 hours after fusion. (B) A metaphase II oocyte fused with an haploid parthenogenetic egg - hybrid arrested in M-phase, observed 24 hours after fusion. (C) Two fused haploid parthenogenetic eggs - hybrid in interphase after cleavage, observed 24 hours after fusion. (D-F) Metaphase II oocytes fused with late fertilized eggs - hybrids going out of M-phase into

anaphase/telophase, observed 2-3 hours after fusion. (G) Metaphase II oocyte fused with a late fertilized egg - hybrid arrested in M-phase, observed 24 hours after fusion. (H) Metaphase II oocyte fused with a late fertilized egg - hybrid in interphase with two nuclei (one out of focus), observed 5 hours after fusion. Bar, $25 \mu \mathrm{m}$.

\section{Oocyte activation is induced after fusion with fertilized eggs}

For fusion of metaphase II oocytes with late zygotes, we used fertilized eggs with a visible nuclei, selected from a population in which some had already undergone nuclear envelope breakdown (NEBD) naturally. This indicated that the population of fertilized eggs had reached the G2 phase of the first mitotic cycle (27-29 hours after hCG). 10-15 minutes after fusion with metaphase II oocytes, the nuclear envelope of the fertilized egg pronuclei broke down in all hybrid cells $(126 / 126)$. However, most $(77 \%)$ of these hybrids did not remain in metaphase (Table 1 , line 5) and by 2 hours after fusion, activated hybrid cells were in anaphase/telophase (Fig. 1D-F). Those hybrids which did not activate were still arrested 
Table 1. Proportion of M-phase arrested hybrids under various experimental conditions

\begin{tabular}{lccc}
\hline Metaphase II oocyte fused with & $\begin{array}{c}\text { M-phase } \\
\text { arrested } \\
\text { hybrids }\end{array}$ & $\begin{array}{c}\text { Total } \\
\text { number } \\
\text { of hybrids }\end{array}$ & $\begin{array}{c}\% \\
\text { Arrested }\end{array}$ \\
\hline 1 Metaphase II & 23 & 27 & 85 \\
2 Haploid parthenogenetic eggs & 36 & 41 & 88 \\
3 Diploid parthenogenetic eggs & 22 & 22 & 100 \\
4 Early zygotes & 11 & 39 & 28 \\
5 Late zygotes & 29 & 126 & 23 \\
6 Early 2-cell embryos & 20 & 45 & 44 \\
7 Late 2-cell embryos & 54 & 62 & 87 \\
8 Zygotes - BAPTA & 6 & 17 & 35 \\
9 Zygotes + BAPTA & 14 & 14 & 100 \\
10 Early 2-cell embryos - BAPTA & 2 & 14 & 14 \\
11 Early 2-cell embryos + BAPTA & 21 & 25 & 84 \\
\hline
\end{tabular}

in M-phase at the end of the 24 hour culture period and contained either one common $(51 \%)$ or two separate $(49 \%)$ spindles (Fig. 1G). The phenotypes of activated hybrids observed 5 to 6 hours after fusion varied greatly with respect to the number of polar bodies extruded and the number of nuclei within the hybrids (Table 2 and Fig. 1H). This diversity probably reflected the number and position of the spindles present in the hybrids after fusion (Table 2). There is a clear tendency for the two separate spindles, present immediately after fusion in the hybrid cells, to move centrally and fuse as has been described for oocytes undergoing the first meiotic division in the presence of cytochalasin D (Kubiak et al., 1991). When the spindles are located centrally, the hybrids often failed to divide after activation, as observed previously in parthenogenetically activated oocytes (Kaufman, 1983; Webb et al., 1986).

\section{Oocyte activation is not dependent upon the nuclear-cytoplasmic ratio of the hybrids}

To determine whether the difference in hybrid cell behavior was dependent upon the amount of nuclear material entering the metaphase II oocyte cytoplasm (diploid (2C) from fertilized eggs versus haploid (1C) from parthenogenetically activated oocytes), we produced hybrid cells in which metaphase II oocytes were fused with diploid parthenogenetically activated oocytes. All of these hybrid cells (22/22) blocked in M-phase, excluding the possibility that the different

Table 2. Morphology of the activated hybrids formed from metaphase II oocytes and late fertilized eggs observed 5-6 hours after fusion

\begin{tabular}{|c|c|c|c|c|c|}
\hline \multicolumn{4}{|c|}{ Morphology of the hybrids } & \multicolumn{2}{|c|}{$\begin{array}{c}\text { Probable configuration } \\
\text { of the spindle(s) } \\
\text { in the hybrids }\end{array}$} \\
\hline $\begin{array}{c}\text { Number of } \\
\text { polar bodies }\end{array}$ & $\begin{array}{l}\text { Number } \\
\text { of nuclei }\end{array}$ & $\begin{array}{c}\text { Number } \\
\text { of hybrids }\end{array}$ & $\begin{array}{c}\% \text { of } \\
\text { hybrids }\end{array}$ & $\begin{array}{c}\text { Number } \\
\text { peripheral }\end{array}$ & $\begin{array}{c}\text { Number } \\
\text { central }\end{array}$ \\
\hline 0 & 2 & 13 & 24 & 0 & 1 \\
\hline 0 & 4 & 8 & 15 & 0 & 2 \\
\hline 1 & 1 & $7 *$ & 13 & 1 & 0 \\
\hline 1 & 3 & 17 & 32 & 1 & 1 \\
\hline 2 & 2 & 7 & 13 & 2 & 0 \\
\hline 2 & 1 & 2 & 3 & 2 & 0 \\
\hline
\end{tabular}

reaction of hybrids was dependent upon the nuclear-cytoplasmic ratio (Table 1 , line 3 ).

\section{Calcium chelation prevents oocyte activation after fusion with fertilized eggs}

The activation of murine oocytes is normally caused by an increase in intracellular calcium concentration within the oocyte (Cuthbertson et al., 1981). We therefore examined whether the same mechanism was responsible for the activation of metaphase II oocytes fused with fertilized eggs. Chelation of intracellular calcium with the calcium chelator BAPTA-AM, immediately after fusion, prevented the fertilized egg inducing the activation of the metaphase II partner in all hybrid cells tested (14/14), whilst the control hybrid cells cultured in the absence of the inhibitor underwent activation (11/17; Table 1, lines 8-9). We confirmed also that BAPTA had no effect on the progression of mitosis by incubating late fertilized eggs with BAPTA-AM. This treatment did not inhibit NEBD, karyokinesis or cytokinesis (data not shown). These observations demonstrate the involvement of calcium in the triggering of oocyte activation by fertilized eggs.

\section{The ability of fertilized embryos to induce oocyte activation disappears with time}

To determine the period during which the ability to destroy CSF is present, we fused metaphase II oocytes with embryos at various stages. When early zygotes (21-24 hours after hCG) were fused with metaphase II oocytes, most of them activated (72\%; Table 1, line 4) confirming that this ability is indeed present in zygotes a few hours after fertilization. When we fused early 2-cell stage embryos in G1/S of the second embryonic cycle with metaphase II oocytes, $56 \%$ of the hybrids activated (Table 1, line 6). The exit from M-phase induced by early 2-cell embryos was also dependent upon calcium release since BAPTA-AM inhibited this transition in $84 \%$ of the hybrids (Table 1, lines 10-11). The incidence of activation was reduced further when late 2-cell stage embryos in G2 of the second embryonic cycle were used. Only $13 \%$ of these hybrid cells activated (Table 1, line 7). To maintain the same volume of cytoplasm in all experimental hybrid cells, both blastomeres of the 2-cell embryo were fused with the metaphase II oocyte. Thus, the ability to destroy CSF is present throughout the first cell cycle, still present in early 2-cell embryos and lost in late 2-cell embryos.

\section{DISCUSSION}

The results presented here demonstrate that fertilized eggs, but not parthenogenetically activated oocytes, are able to induce the destruction of the MPF and CSF activities found in metaphase II arrested mouse oocytes. Since this ability is not due to the double amount of chromatin present in fertilized eggs, as shown by the fact that hybrids made up of metaphase II oocytes and diploid parthenogenetically activated eggs arrest in M-phase, it must be linked to fertilization and thus comes from the sperm. We observed also that this ability to destroy MPF and CSF activities, present in fertilized 1-cell and early 2-cell stage embryos, is lost in late 2-cell stage embryos. This suggests that the inactivation of this mechanism is not linked to the passage through a mitotic M-phase. Finally, this ability 
is dependent upon calcium release, like in the normal situation at fertilization, since it can be blocked by BAPTA-AM.

It is well known that in mammals the sperm activates the oocyte by inducing multiple intracellular free calcium transients at fertilization (Cuthbertson et al., 1981; Cuthbertson and Cobbold, 1985; Kline and Kline, 1992). The effect of calcium transients on MPF and CSF destruction is still unclear. It is known that, in Xenopus oocytes, the calcium peak at fertilization activates transiently the calmodulin dependent protein kinase II which itself, through unknown mechanisms, activates the cyclin degradation machinery (Lorca et al., 1993). This activation of the cyclin degradation machinery is also short-lived, as demonstrated in mouse oocytes (Winston et al., 1994). The major component of CSF, the c-mos gene product, is degraded when MPF has already been inactivated in both activated Xenopus and mouse eggs (Lorca et al., 1991; Watanabe et al., 1991; Weber et al., 1991), by a mechanism involving the ubiquitin dependent pathway (Ishida et al., 1993).

The mechanism by which sperm stimulates the calcium oscillations is still controversial, there being two different hypotheses put forward. One proposes that the sperm binds to a membrane receptor coupled to GTP binding proteins, which in turn stimulate phosphoinositide turnover and increase the production of the intracellular calcium-releasing second messenger, inositol-1,4,5-triphosphate (InsP3; Miyazaki, 1988). The other suggests that the sperm triggers calcium release by fusing with the egg and introducing a cytoplasmic factor (Swann and Whitaker, 1990; Tesarik and Mendoza, 1994). Swann (1990) has shown that cytosolic sperm proteins injected into the hamster oocyte generate repetitive calcium oscillations similar to those observed at fertilization. Our observations demonstrate that the ability to destroy MPF and CSF activities is still present in the fertilized egg 12-14 hours post-fertilization, while calcium transients seem to stop 3 to 4 hours after fertilization (Kline and Kline, 1992). If this ability was due to an interaction of the sperm with an oocyte receptor, this would suggest that underlying oscillations, for example of InsP3 (Ciapa et al., 1994) that are able to drive calcium transients, are still taking place at the end of the first cell cycle. However, should the calcium stores become depleted in the egg a few hours after fertilization, calcium transients are no longer observed until new calcium stores, brought by fusion with the metaphase II oocytes, are available. Alternatively, the sperm may bring a component able to trigger the calcium transients into the oocyte that becomes unable to induce calcium transients in zygotes a few hours after fertilization, due either to its sequestration within a subcellular domain or to the lack of available calcium stores. Its introduction into a metaphase II oocyte (by fusion with a fertilized egg that contains this sperm component) would be able to trigger again the mechanisms involved in calcium release. From our data, it is not possible to distinguish between these two possibilities.

Finally, CSF was discovered in frog eggs by transferring cytoplasm from metaphase II arrested oocytes to blastomeres at the 2-cell stage (Masui and Markert, 1971). This 'CSF assay' has been used widely (see for example Watanabe et al., 1991; Haccard et al., 1993). However, no data is available concerning cytoplasmic transfer into amphibian zygotes (1-cell stage). It is not unlikely therefore, that the ability to destroy CSF may be present until the 2-cell stage in embryos from other vertebrates, including frogs.
We thank D. Maluchnik and N. Winston for critical reading of the manuscript and R. Schwartzmann and G. Géraud for their expert photographic work. We are grateful to Dr J. Kilmartin for the gift of the YL1/2 antibody. This work was supported by grants from La Ligue contre le Cancer, l'Association pour la Recherche contre le Cancer and the Faculty of Biology of the University of Warsaw.

\section{REFERENCES}

Ciapa, B., Pesando, D., Wilding, M. and Whitaker, M. (1994). Cell-cycle calcium transients driven by cyclic changes in inositol trisphosphate levels. Nature 368, 875-878.

Cuthbertson, K. S. R., Wittingham, D. G. and Cobbold, P. M. (1981). Free $\mathrm{Ca}^{++}$increases in exponential phases during mouse oocyte activation. Nature 294, 754-757.

Cuthbertson, K. S. R. (1983). Parthenogenetic activation of mouse oocytes in vitro with ethanol and benzyl alcohol. J. Exp. Zool. 226, 311-314.

Cuthbertson, K. S. R. and Cobbold, P. M. (1985). Phorbol ester and sperm activate mouse oocyte by inducing sustained oscillations in cell $\mathrm{Ca}^{++}$. Nature 316, 541-542.

Gabrielli, B. G., Roy, L. M. and Maller, J. L. (1993). Requirement for cdk2 in cytostatic factor-mediated metaphase arrest. Science 259, 17661769.

Haccard, O., Sarcevic, B., Lewellyn, A., Hartley, R., Roy, L., Izumi, T., Erikson, E. and Maller, J. L. (1993). Induction of metaphase arrest in cleaving Xenopus embryos by MAP kinase. Science 262, 1262-1265.

Ishida, N., Tanaka, K., Tamura, T., Nishizawa, M., Okazaki, K., Sagata, N. and Ichihara, A. (1993). MOS is degraded by the $26 \mathrm{~S}$ proteasome in a ubiquitin-dependent fashion. FEBS Lett. 324, 345-348.

Kaufman, M. H. (1983). Early Mammalian Development: Parthenogenetic Studies. Cambridge: Cambridge University Press.

Kline, D. and Kline, J. T. (1992). Repetitive calcium transients and the role of calcium in exocytosis and cell cycle activation in the mouse egg. Dev. Biol. 149, 80-89.

Kubiak, J. Z. (1989). Mouse oocytes gradually develop the capacity for activation during the metaphase II arrest. Dev. Biol. 136, 537-545.

Kubiak, J. Z., Paldi, A., Weber, M. and Maro, B. (1991). Genetically identical parthenogenetic mouse embryos produced by inhibition of the first meiotic division by cytochalasin D. Development 111, 763-770.

Kubiak, J. Z., Weber, M., de Pennart, H., Winston, N. and Maro, B. (1993). The metaphase II arrest in mouse oocytes is controlled through microtubuledependent destruction of cyclin B in the presence of CSF. EMBO J. 12, 37733778 .

Lorca, T., Galas, S., Fesquet, D., Devault, A., Cavadore, J.-C. and Dorée, M. (1991). Degradation of the proto-oncogene product p39mos is not necessary for cyclin proteolysis and exit from meiotic metaphase: requirement for a $\mathrm{Ca}^{2+}$-calmodulin dependent event. EMBO J. 10, 20872093.

Lorca, T., Cruzalequi, F. H., Fesquet, D., Cavadore, J.-C., Méry, J., Means, and Dorée, M. (1993). Calmodulin-dependent protein kinase II mediates $\mathrm{Ca}^{2+}$-dependent inactivation of MPF and CSF activities upon the fertilization of Xenopus eggs. Nature 366, 270-273.

Maro, B., Johnson, M. H., Pickering, S. J. and Flach, G. (1984). Changes in the actin distribution during fertilisation of the mouse egg. J. Embryol. Exp. Morph. 81, 211-237.

Masui, Y. (1991). The role of Cytostatic Factor (CSF) in the control of oocyte cell cycles - a summary of 20 years of study. Dev. Growth Differ. 33, 543551 .

Masui, Y. and Markert, C. L. (1971). Cytoplasmic control of nuclear behavior during meiotic maturation of frog oocytes. J. Exp. Zool. 117, 129146.

Miyazaki, S. (1988). Inositol 1,4,5-triphosphate-induced calcium release and guanine nucleotide-binding protein-mediated periodic calcium rises in golden hamster eggs. J. Cell Biol. 106, 345-353.

Norbury, C. and Nurse, P. (1992). Animal cell cycles and their control. Annu. Rev. Biochem. 61, 441-470.

Sagata, N., Watanabe, N., Van de Woude, G. F. and Ikawa, Y. (1989). The c-mos proto-oncogene product is a cytostatic factor responsible for meiotic arrest in vertebrate eggs. Nature 342, 512-518.

Spindle, A. (1981). Polyethylene glycol-induced fusion of two-cell mouse embryo blastomeres. Exp. Cell Res. 131, 465-470.

Swann, K. (1990). A cytosolic sperm factor stimulates repetitive calcium 


\section{4}

\section{Zernicka-Goetz and others}

increases and mimics fertilization in hamster eggs. Development 110, 1295 1302.

Swann, K. and Whitaker, M. J. (1990). Second messengers at fertilization in sea-urchin eggs. J. Reprod. Fert. 42 (suppl.), 141-53.

Tarkowski, A. K. (1966). An air-drying method for chromosome preparation from mouse eggs. Cytogenetics 5, 394-400.

Tesarik, J. and Mendoza, C. (1994). Most living acrosome-reacted spermatozoa do not fuse with the oocyte when inserted into the perivitelline space. Fertil. Steril. 61, 529-535.

Vincent, C., Cheek, T. R. and Johnson, M. H. (1992). Cell cycle progression of parthenogenetically activated mouse oocytes to interphase is dependent on the level of internal calcium. J. Cell Sci. 103, 389-396.

Watanabe, N., Hunt, T., Ikawa, Y. and Sagata, N. (1991). Independent inactivation of MPF and cytostatic factor (Mos) upon fertilization of Xenopus eggs. Nature 352, 247-248.

Webb, M., Howlett, S. K. and Maro, B. (1986). Parthenogenesis and cytoskeleton organisation in ageing mouse oocytes. J. Embryol. Exp. Morph. 95, 131-145.

Weber, M., Kubiak, J. Z., Arlinghaus, R. B., Pines, J. and Maro, B. (1991). c-mos proto-oncogene product is partly degraded after release from meiotic arrest and persists during interphase in mouse zygotes. Dev. Biol. 148, 393 397.

Whittingham, D. G. (1971). Culture of mouse ova. J. Reprod. Fert. 14 (suppl.), 7-21.

Winston, N. J., McGuinness, O., Johnson, M. H. and Maro, B. (1995). The exit of mouse oocytes from meiotic M-phase requires an intact spindle during intracellular calcium release. J. Cell Sci. 108, 143-151.

Zernicka-Goetz, M. (1991). Spontaneous and induced activation of rat oocytes. Mol. Reprod. Dev. 28, 169-176.

(Received 14 October 1994 - Accepted 15 November 1994) 